\title{
Prevalence of Shiga toxin-producing and Enteropathogenic Escherichia coli Isolated from Chicken Meat in the west of Iran
}

omid zarei

Tehran University of Medical Sciences

Leili Shokoohizadeh

Hamadan University of Medical Sciences Medical School

Hadi Hossainpour

Hamadan University of Medical Sciences Medical School

Mohammad Yousef Alikhani ( $\square$ alikhani43@yahoo.com )

https://orcid.org/0000-0003-4577-4029

Research note

Keywords: E. coli, STEC, EPEC, Shiga toxin, Poultry meat

Posted Date: December 19th, 2019

DOI: https://doi.org/10.21203/rs.2.11448/v4

License: (c) (i) This work is licensed under a Creative Commons Attribution 4.0 International License.

Read Full License 


\section{Abstract}

Objective: Shiga toxin-producing Escherichia coli (STEC) is known as a crucial zoonotic foodborne pathogen. Totally, 257 raw chicken meat were collected from markets in Hamadan, west of Iran. The samples were cultured on selective media and the virulence genes of E. coli isolates were analyzed by PCR. The antibiotic resistance patterns were determined by the disk diffusion method. The genetic relatedness of the E. coli 0157 isolates was analyzed by ERIC-PCR. Results: Totally, 93 (36\%; 95\% Cl 41.9$30.1 \%$ ) isolates were identified as E. coli. Based on microbiological tests, 36 (38.7\%; 95\% Cl 48.6-28.8), 7 (7.5\%; 95\% $\mathrm{Cl} 12.8-2.2 \%)$, and 12 (12.9\%; $95 \% \mathrm{Cl} 19.7-6.1 \%)$ of the E. coli isolates were characterized as STEC, Enteropathogenic E. coli, and attaching and effacing E. coli (AEEC) strains, respectively. A high level of resistance to nalidixic acid (91.4\%; 95\% Cl 97.1- 85.7\%), tetracycline (89.8\%; 95\% Cl 96.2-83.5\%), ampicillin (82.8\%; 95\% Cl 90.2-75.1\%), and sulfametoxazole-trimotoprime (71\%; 95\% Cl 80.2-61.8\%) was detected among the E. coli isolates. The analysis of ERIC-PCR results showed five different ERIC types among the E. coli 0157 isolates. Based on findings. Control and check-up of poultry meats should be considered as a crucial issue for public health.

\section{Introduction}

Diarrheagenic E. coli, which causes diarrhea in humans, can be classified into seven different pathotypes on the basis of its specific virulence properties, distinct epidemiology, and clinical features:

Enterotoxigenic E. coli (ETEC), Enteroinvasive E. coli (EIEC), Enteroaggregative E. coli (EAEC), DiffuseAdhering E. coli (DAEC), Cytolethal distending toxin-producing E. coli, Enteropathogenic E. coli (EPEC), and Shiga toxin-producing E. coli (STEC) [1]. STECs are one of the most important pathogens transmitted by food. In addition to causing food poisoning, these strains can cause severe diseases such as diarrhea, bleeding colitis, hemolytic uremic syndrome, thrombocytopenic purpura, and death. Most cases of ulcerative colitis and hemolytic uremic syndrome are related to the 0157:H7 serotype which is considered as the most important serotype of this strain. Several outbreaks of bacterial foodborne disease due to the consumption of undercooked or raw meat contaminated with STEC strains have been reported $[1,2]$.

In addition to Shiga toxins, an external membrane protein called intimin is responsible for the attachment of bacteria to the intestinal epithelial cells, causes a certain damage (attaching-effacing lesions (A/E)), and is encoded by the eae gene [3, 4]. Also, enterohemolysin, encoded by the $h l y$ gene, is an effective factor in the pathogenicity of STEC [5]. Because only limited and incomplete studies have been conducted on the prevalence and epidemiology of the 0157:H7 serotype in developing countries, its prevalence has been reported as low $[6,7]$.

The EPEC pathovar plays an important role as a causative agent of infantile diarrhea in developing countries [8]. This pathovar has intimin which is encoded by the chromosomal eae gene. It also possesses the ability to form A/E lesions on intestinal cells but does not contain Shiga toxin-encoding genes. 
Attaching and effacing E. coli (AEEC) are characterized by their ability to cause attaching and effacing (A/E) lesions in the gut mucosa of human and animal hosts leading to diarrhea. Thus, two groups of $E$. coli strains that cause attaching and effacing (A/E) lesions are classified as AEEC. In Iran, most molecular studies on the STEC have been done on dairy and animal stool samples and little information is available on STEC and EPEC strains from poultry sources. The aim of this study was to detect the virulence factors $s t x 1$, stx2, eae, and $h l y A$ in the $E$. coli isolates and also to perform molecular typing of 0157:H7 strains isolated from raw chicken meat samples.

\section{Methods}

\section{Identification of $E$. coli strains}

In this cross-sectional study, 257 raw chicken meat samples were randomly collected using an electronic random number generator (www.randomresult.com) from different butchers and supermarkets of different area of Hamadan city, west of Iran, from January 2016 to May 2017. The samples were transferred to sterile tubes containing thioglycolate broth media after homogenization, and incubated overnight at $37^{\circ} \mathrm{C}$. They were inoculated on MacConkey agar plates (Merck, Germany) and incubated at $37^{\circ} \mathrm{C}$ for $24 \mathrm{~h}$. The E. colilike colonies were subjected to different biochemical tests including sugar fermentation, Simmons' citrate, indole production, motility, methyl red, and Voges-Proskauer (IMVIC) tests [1]. The sorbitol MacConkey agar (Merck, Germany) and serogrouping with anti-0157 sera (Baharafshan, Iran) were used for the diagnosis of the E. coli 0157 serotype.

\section{Antimicrobial susceptibility testing}

The antimicrobial susceptibility of $E$. coli isolates to cefotaxime (CTX), ceftazidime (CAZ), cefepime (CPM), cefoxitin (FOX), cefexime (CFM), nitrofurantoin (NIT), gentamicin (GEM), ciprofloxacin (CIP), nalidixic acid (NA), sulfamethoxazole-trimethoprim (SXT), aztreonam (ATM), amoxicillin (AMX), ampicillin (AMP), tetracycline (TET), minocycline (MIN), and imipenem (IPM) was detected by the disk diffusion method according to CLSI guidelines [9].

\section{Detection of virulence genes}

A sweep of five $E$. coli colonies on MacConkey agar was inoculated in LB broth and incubated overnight at $37^{\circ} \mathrm{C}$ and the genomic DNAs of the colonies were extracted by boiling method [10]. The virulence genes st 1, st 2 , $h l y A$, and eae were detected by PCR method using the primers described in previous studies [11].

The virulence genes eae, st $x$, and $s t x 2$ were detected using a triplex PCR in a reaction mixture with a total volume of $20 \mu \mathrm{L}$, included $10 \mu \mathrm{L}$ PCR Master Mix 2x (Fermentas, Lithuania), $6 \mu \mathrm{L}$ double distilled water, $1 \mu \mathrm{L}$ from each primers, and $2 \mu \mathrm{L}$ DNA template. The cycling program was used as follows: initial denaturation ( $3 \mathrm{~min}$ at $\left.94^{\circ} \mathrm{C}\right)$, followed by 35 cycles of denaturation $\left(1 \mathrm{~min}\right.$ at $\left.94^{\circ} \mathrm{C}\right)$, annealing $(1 \mathrm{~min}$ at $\left.55^{\circ} \mathrm{C}\right)$, extension $\left(1 \mathrm{~min}\right.$ at $\left.72^{\circ} \mathrm{C}\right)$, and final extension $\left(7 \mathrm{~min}\right.$ at $\left.72{ }^{\circ} \mathrm{C}\right)$. For the $h / y$ gene, a single $\mathrm{PCR}$ 
reaction was done with the same conditions as mentioned above except that annealing was at $63^{\circ} \mathrm{C}$ for $1 \mathrm{~min}$.

\section{ERIC-PCR of E. coli 0157 isolates}

For molecular typing and detection of the genetic linkage among E. coli 0157 serotypes, ERIC-PCR was carried out using ERIC primers and the conditions described in a previous study [12]. The banding patterns of ERIC were analyzed by an online data analysis service (inslico.ehu.es). The ERIC profiles were compared by the Dice method and were clustered by the UPGMA program.

\section{Results And Discussion}

Among the 257 raw poultry samples, $93(36 \% ; 95 \% \mathrm{Cl} 41.9-30.1 \%)$ isolates were identified as $E$. coli. Based on serological and microbiological tests, 36 (38.7\%; 95\% Cl 48.6-28.8), 7 (7.5\%; 95\% Cl 12.8-2.2\%), and 12 (12.9\%; $95 \% \mathrm{Cl} 19.7-6.1 \%)$ E. coli isolates were characterized as STEC (st $x 1^{+}$and/or $s t \times 2^{+}$and eae+/ eae-), EPEC (eae $e^{+}$, and AEEC strains (EPECs and eae+ strains of STECs), respectively. All of the STEC isolates showed colorless colonies on the sorbitol MacConkey media.

The results of the antimicrobial susceptibility test conducted on $93 \mathrm{E}$. coli isolates are shown in Figure 1. Based on the results, all of the isolates $(100 \%)$ were susceptible to cefotaxime, cefoxitin, ceftazidime, and aztreonam. A high-level of resistance to nalidixic acid (91.4\%; $95 \% \mathrm{Cl}$ 97.1- 85.7\%), tetracycline (89.8\%; 95\% Cl 96.2-83.5\%), ampicillin (82.8\%; 95\% Cl 90.2-75.1\%), and sulfamethoxazole-trimethoprim (71\%; $95 \% \mathrm{Cl} 80.2-61.8 \%)$ was detected among the $E$. coli isolates. The PCR results showed that the distribution of the virulence genes $s t \times 1$, st $\times 2$, and eae among the $93 \mathrm{E}$. coli isolates was 15 (16.1\%; $95 \% \mathrm{Cl} 23.6-8.6 \%)$, 31 (33.3\%; 95\% Cl 42.9-23.7\%), and 12 (12.9\%; 95\% Cl 19.7-6.1\%), respectively. All of E. coli 0157 strains showed $s t \times 1^{+} / s t \times 2^{+} / e a e^{+}\left(1\right.$ isolate), st $x 1^{+} / \mathrm{eae}^{+}$(2 isolates), and $s t \times 2^{+} / e a e^{+}$( 2 isolates) patterns. The hlyA gene was not detected in any of the $E$. coli isolates (Figure 2). The analysis of the ERIC-PCR results showed genetic diversity among $E$. coli 0157 strains because five different ERIC patterns were observed among these strains (Figure 3).

The results shown that chicken meat can be contaminated with $E$. coli. This organism was isolated from $93(36 \%)$ raw chicken meat samples and $36(38.7 \%), 7(7.5 \%)$, and $12(12.9 \%)$ of the $E$. coli isolates were characterized as STEC, EPEC, and AEEC strains. The st $x 2$ gene was the most frequent virulence factor among the STEC isolates. The major animal source of STEC is primarily cattle, followed by sheep, goats, pigs, and poultry. Poultry meat is known as the potential source of STEC contamination compared to other sources of meat. In Korea, STEC was isolated in $22.6 \%$ of beef, $7.3 \%$ of poultry, and $2.0 \%$ of pork meat samples [13]. In the current study, 0157 E. coli isolates having stx 1 and/or stx2 and eae were detected in $5.3 \%$ of the poultry meat samples and recognized as STEC strains. Although the prevalence of this isolate was not significant, this rate of infection is considerable from the public health point of view.

The prevalence of STEC and AEEC in the current study is different from that of some studies in Iran and other countries. In the current study, higher STEC and lower AEEC isolates were detected compared to the 
study of Momtaz el al. They reported that the prevalence of STEC and AEEC were $21 \%$ and $34 \%$, respectively [14]. They also reported that stx 1 was the most frequent (96\%) virulence factor among the isolates. In contrast, in the current study, stx 1 was found only in $16 \%$ of the isolates. One of the reasons for this difference in frequency can be the difference in the number of samples studied. However, Guran et al. showed that the overall prevalence of E. coli 0157 in poultry meat samples collected from supermarkets in Diyarbakir, Turkey was 1.3\% [15]. One of the significant results of the current study is that $12.9 \%$ of the $E$. coli isolates were identified as AEEC. Intimin genes are present in EPEC and in some STEC. Atypical EPEC or AEEC appears to be more closely related to STEC [15-17]. Based on the results of the current study, the role of AEEC strains in gastrointestinal infection needs further investigations.

In this study, the E. coli 0157 strains were positive for stx1, stx2, and eae genes. In India, Dutta et al. reported that $14(33.33 \%)$ isolates carried at least 1 virulence genes and $10(23.81 \%)$ of these isolates (collected from poultry samples) were recorded as STEC and 4 (9.52\%) of them were recorded as EPEC [18].

In a review study, the resistance rates of $E$. coli strains to tetracycline, sulfamethoxazole, streptomycin, and ampicillin were more than $40 \%$ in all the studied countries. Increasing antibiotic resistance is a major concern for animal and human health because of the high consumption of antibiotics in veterinary medicine. Resistant bacteria can spread from food-producing animals to humans. The information from the evaluated countries indicates that such antibiotics are usually used in poultry industry [19].

In this study, the resistance levels of STEC to some antimicrobial agents such as nalidixic acid, ampicillin, tetracycline, and trimethoprim-sulfamethoxazole ranged from 71 to $91 \%$. According to these results, the poultry meat contaminated with STEC strains can be a potential source of antimicrobial resistance.

Momtaz et al. reported the high resistance of STEC strains to tetracycline, chloramphenicol, and nitrofurantoin (63 to 77\%). According to our findings and studies by others, the prescription of tetracycline is recommended neither in cases of $E$. coli infection nor in veterinary medicine with respect to poultry products [14]. There are few reports about the molecular typing of STECs from poultry sources in Iran and other countries. In the current study, ERIC-PCR genotyping demonstrated 5 different ERIC-genotypes from 5 E. coli 0157 isolates. Therefore, the results of the current study showed genetic diversity among $E$. coli 0157 isolates as well as the different potential sources of E. coli 0157 contamination. The results also indicated the usefulness of the PCR-based genotyping method in the epidemiological investigations of virulent E. coli strains. Consistent with our results, in a study by Sekhar et al. in India, the ERIC-PCR results discriminated 12 STEC isolates from poultry samples into 11 ERIC-PCR genotypes [20].

In conclusion, the results of the current study revealed that poultry meat can be considered as a source of pathogenic E. coli strains. Pathogenic E. coli strains in poultry meat samples were detected by such accurate and quick techniques as PCR assay. The detection of STEC (38\%) was a significant finding. The stX2 was identified as the most frequent virulence factor among the STEC isolates. Our results indicate the need for more attention to poultry meat control, antibiotic administration in veterinarians and $E$. coli 
virulence genes, especially st 1 , st $x 2$ and eae, which are largely present in pathogenic $E$. coli strains isolated from poultry meat.

\section{Limitations}

One of the most important limitations of this study was the rather few number of raw poultry meat samples. More samples are required for such molecular studies. We also had some limitations in financial support for obtaining information about poultry raising systems and slaughter systems to discuss the sources of contamination by robust typing methods.

\section{Abbreviations}

E. coli: Escherichia coli

STEC: Shiga toxin-producing Escherichia coli

AEEC: Attaching and effacing Escherichia coli

EPEC: Enteropathogenic Escherichia coli

Eae. Escherichia coli attaching and effacing

stx1 and stx2: Shiga toxin 1 and 2.

hly. Hemolysin

PCR: Polymerase chain reaction

\section{Declarations}

\section{Authors' contributions}

$\mathrm{OZ}, \mathrm{HH}$, and MA conceived the study. $\mathrm{OZ}$ and LS conducted the experiments and analyzed the results. $\mathrm{OZ}$ and LS drafted the manuscript and made substantial contributions to the design of the study. OZ, MA, and LS critically reviewed the manuscript. OZ and LS participated in data analysis. All authors read and approved the final manuscript.

\section{Authors' details}

${ }^{1}$ Student Research Committee, Hamadan University of Medical Sciences, Hamadan, Iran.

2 Department of Microbiology, Faculty of Medicine, Hamadan University of Medical Sciences, P.O box: 6517838678, Hamadan, Iran.

\section{Acknowledgements}


We would like to thank all members of the microbiology laboratory of Hamadan University of Medical Sciences.

\section{Competing interests}

The authors declare that they have no competing interests.

\section{Availability of data and materials}

All the information supporting our conclusions and appropriate references are included in the manuscript.

\section{Consent for publication}

Not applicable.

\section{Ethics approval and consent to participate}

The present study was ethically approved by the Institutional Review Board of Hamadan University of Medical Sciences (IR.UMSHA.REC.1398.12).

\section{Funding}

This research was funded by a grant from the Student Research Center of Hamadan University of Medical Sciences, Hamadan, Iran.

\section{References}

1. Bouzari S, Farhang E, Hosseini SM, Alikhani MY: Prevalence and antimicrobial resistance of shiga toxin-producing Escherichia coli and enteropathogenic Escherichia coli isolated from patients with acute diarrhea. Iranian journal of microbiology 2018, 10(3):151.

2. Dehkordi FS, Parsaei P, Saberian S, Moshkelani S, Hajshafiei P, Hoseini S, Babaei M, Ghorbani M: PREVALENCE STUDY OF THEILERIA ANNULATA BY COMPARISON OF FOUR DIAGNOSTIC T. Bulgarian Journal of Veterinary Medicine 2012, 15(2).

3. Momtaz H, Davood Rahimian M, Safarpoor Dehkordi F: Identification and characterization of Yersinia enterocolitica isolated from raw chicken meat based on molecular and biological techniques. Journal of Applied Poultry Research 2013, 22(1):137-145.

4. Exeni RA, Fernandez-Brando RJ, Santiago AP, Fiorentino GA, Exeni AM, Ramos MV, Palermo MS: Pathogenic role of inflammatory response during Shiga toxin-associated hemolytic uremic syndrome (HUS). Pediatric Nephrology 2018, 33(11):2057-2071.

5. Smith J, Fratamico P: Escherichia coli as a Pathogen. In: Foodborne Diseases. Elsevier; 2017: 189208.

6. Pasquali F, Palma F, Trevisani M, Parisi A, Lucchi A, De Cesare A, Manfreda G: Whole genome sequencing based typing and characterisation of Shiga-toxin producing Escherichia coli strains 
belonging to 0157 and 026 serotypes and isolated in dairy farms. Italian Journal of Food Safety 2018, 7(4).

7. Parreira V, Arns $C$, Yano T: Virulence factors of avian Escherichia coli associated with swollen head syndrome. Avian Pathology 1998, 27(2):148-154.

8. Aslani M, Alikhani M: Serotypes of enteropathogenic Escherichia coli isolated from children under 5 years of age. Iranian Journal of Public Health 2009:70-77.

9. CLSI;: Performance standards for antimicrobial susceptibility testing. CLSI document M100-S25 Wayne 2018.

10. Zamani A, Mashouf RY, Namvar AME, Alikhani MY: Detection of magA Gene in Klebsiella spp. Isolated from clinical samplesdetection of magA. Iranian journal of basic medical sciences 2013, 16(2):173.

11. Fagan PK, Hornitzky MA, Bettelheim KA, Djordjevic SP: Detection of Shiga-like toxin (stx1 andstx2), intimin (eaeA), and enterohemorrhagic Escherichia coli (EHEC) hemolysin (EHEC hlyA) genes in animal feces by multiplex PCR. Appl Environ Microbiol 1999, 65(2):868-872.

12. Zarei $\mathrm{O}$, Shokoohizadeh L, Hossainpour H, Alikhani MY: Molecular analysis of Pseudomonas aeruginosa isolated from clinical, environmental and cockroach sources by ERIC-PCR. BMC research notes 2018, 11(1):668.

13. Lee GY, Jang HI, Hwang IG, Rhee MS: Prevalence and classification of pathogenic Escherichia coli isolated from fresh beef, poultry, and pork in Korea. International journal of food microbiology 2009, 134(3):196-200.

14. Momtaz H, Jamshidi A: Shiga toxin-producing Escherichia coli isolated from chicken meat in Iran: Serogroups, virulence factors, and antimicrobial resistance properties. Poultry science 2013, 92(5):1305-1313.

15. Guran HS, Vural A, Erkan ME, Durmusoglu H: Prevalence and some virulence genes of Escherichia coli 0157 isolated from chicken meats and giblets. Annals of animal science 2017, 17(2):555-563.

16. Fröhlicher E, Krause G, Zweifel C, Beutin L, Stephan R: Characterization of attaching and effacing Escherichia coli (AEEC) isolated from pigs and sheep. BMC microbiology 2008, 8(1):144.

17. Trabulsi LR, Keller R, Gomes TAT: Typical and Atypical Enteropathogenic Escherichia coli. Emerging infectious diseases 2002, 8(5):508.

18. T.K. Dutta PR, S. Bandyopadhyay, S.A. Wani, and I. Hussain: Detection \& characterization of Shiga toxin producing Escherichia coli (STEC) \& enteropathogenic Escherichia coli (EPEC) in poultry birds with diarrhoea. Indian J Med Res 2011 May, 133(5):541-545.

19. N Roth AK, S Mayrhofer, $U$ Zitz: The application of antibiotics in broiler production and the resulting antibiotic resistance in Escherichia coli: A global overview. The application of antibiotics in broiler production and the resulting antibiotic resistance in Escherichia coli: A global overview 2018, 98(4):1791-1804.

20. M. Soma Sekhar MS, T. Srinivasa Rao, and M. Metta: Genotyping of virulent Escherichia coli obtained from poultry and poultry farm workers using enterobacterial repetitive intergenic 
consensus-polymerase chain reaction. http://wwwveterinaryworldorg/2017, 10(11):1292-1296.

\section{Figures}

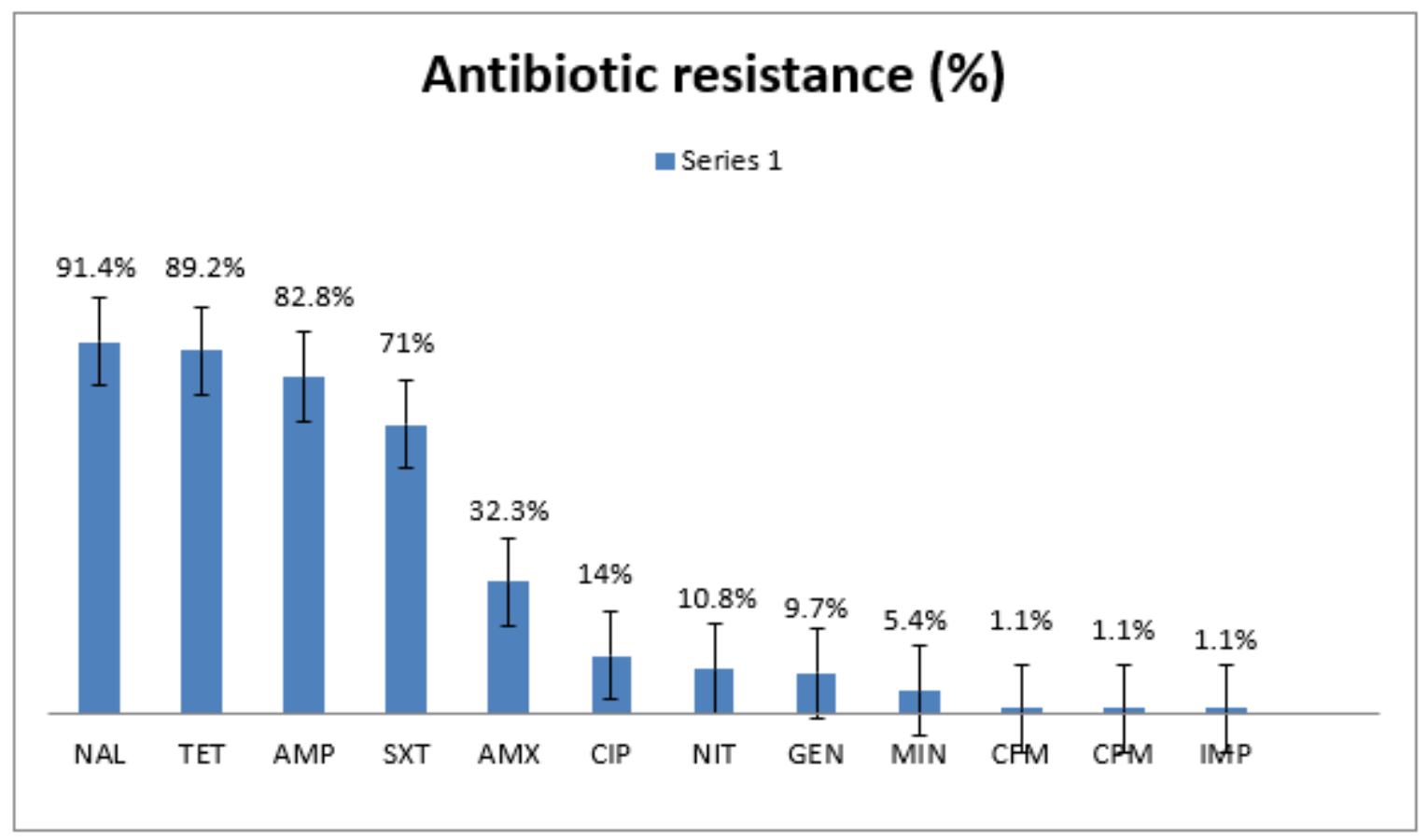

Figure 1

Antibiotic resistance (\%) of E. coli isolated from raw chicken meats NA: nalidixic acid, TET: tetracycline, AMP: ampicillin, SXT: sulfamethoxazole-trimethoprim, AMX: amoxicillin, CIP: ciprofloxacin, NIT: nitrofurantoin, GEM: gentamicin, MIN: minocycline, CFM: cefexime, CPM: cefepime, IPM: imipenem.

\section{Prevalence (\%) of virulence genes}

\section{m virulence genes}

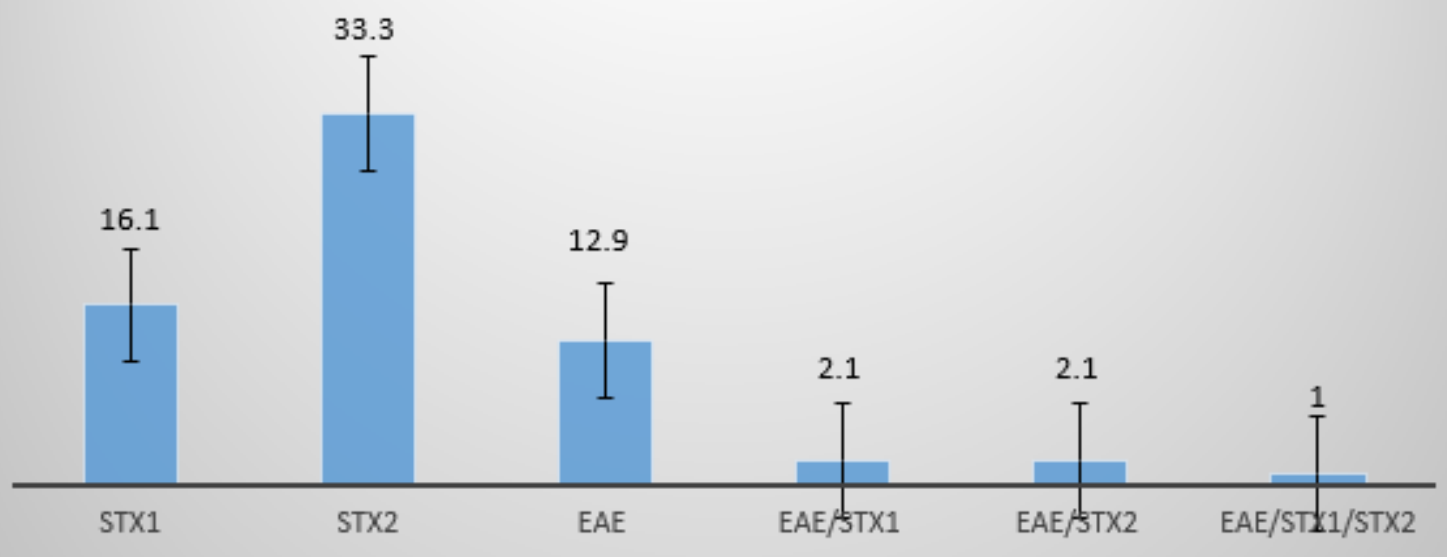


Figure 2

prevalence of virulence genes among $93 \mathrm{E}$. coli strains isolated from raw meat chicken

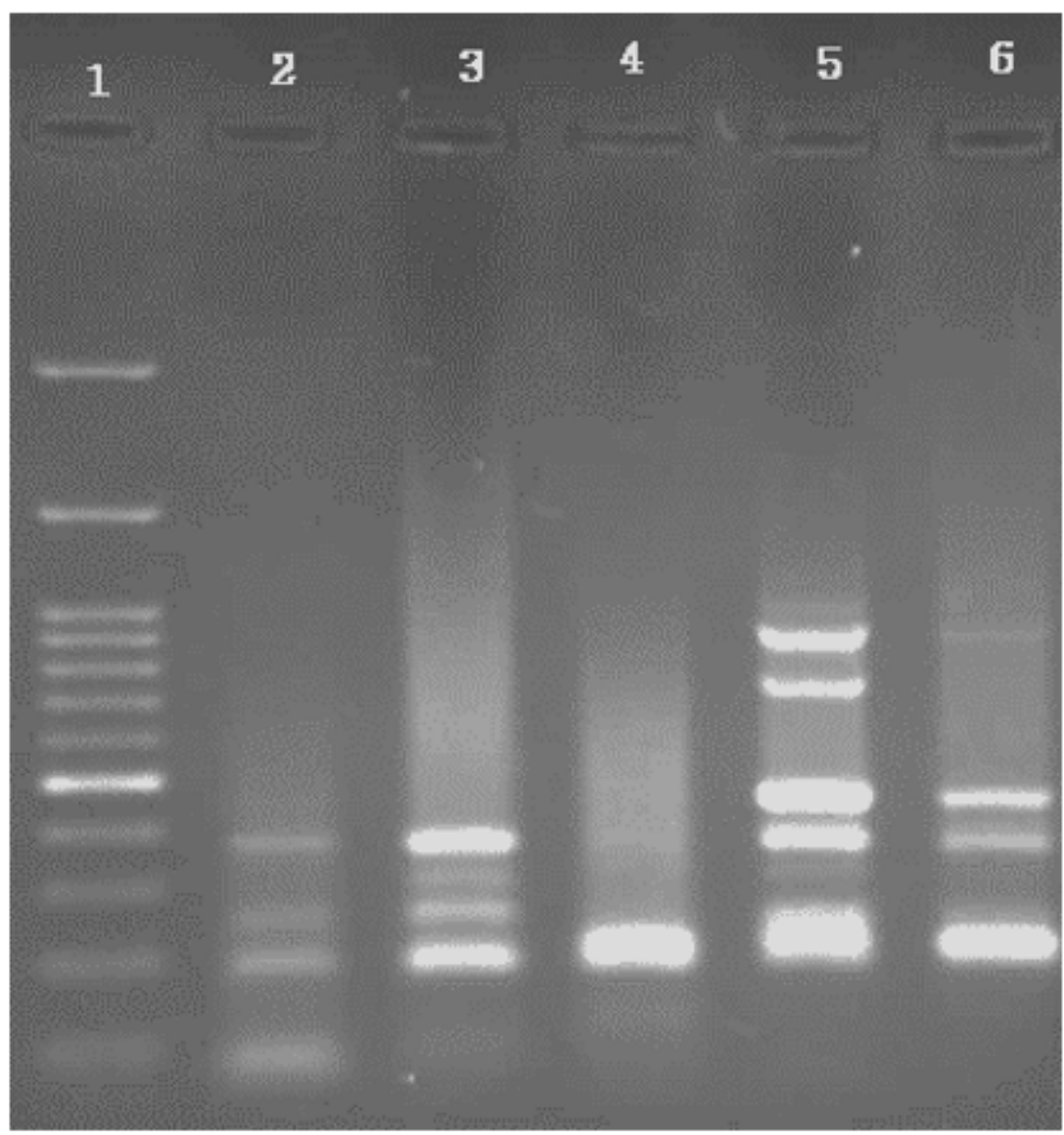

Figure 3

ERIC patterns of 5 different E. coli 0157 isolates Lane 1: ladder 100 bp, lane 2-6: E. coli 0157 